Saudi Journal of Pathology and Microbiology

Abbreviated Key Title: Saudi J Pathol Microbiol ISSN 2518-3362 (Print) IISSN 2518-3370 (Online) Scholars Middle East Publishers, Dubai, United Arab Emirates

Journal homepage: http://scholarsmepub.com/sjpm/

\title{
To Evaluate the Sensitivity of Rosner's Index (ICA) Vs Standard Normalised Ratio in the Interpretation of Mixing Studies in Lupus Anticoagulant
}

\author{
Mirza Asif Baig ${ }^{1 *}$, Syeda Sarah Mahjabeen ${ }^{2}$ Dr Mohd Iqbal Ahmed ${ }^{3}$ \\ ${ }^{1}$ Former Assistant Professor, Dept. of Pathology, Shri B. M. Patil Medical College, Hospital \& Research centre, Bijapur, Karnataka, India \\ ${ }^{2}$ Consultant Hematologist, Medilab Hyderabad, AP, India \\ ${ }^{2}$ Consultant Pediatrician Medicare Children hospital, Hyderabad, India
}

DOI: $\underline{10.36348 / \text { sjpm.2019.v04i11.004 }}$

| Received: 06.11.2019 | Accepted: 13.11.2019 | Published: 15.11.2019

*Corresponding author: Mirza Asif Baig

\section{Abstract}

Introduction: The Current 3 major LAC guidelines (BSH, ISTH \& CLSI) recommends mixing tests for detection of LAC, even though these test order/ sequence vary \& there are certain limitations, but still these guidelines advocates mixing test so as to maximize the diagnostic performance. The main objective of this study is to assess sensitivity of these tests in 255 LAC cases. Results: The Coagulometer used is Sysmex CS-5100. RI Cut offs $\leq 10=$ Correction $\& \geq 15$ $=$ Inhibitor \& SNR > 1.15 indicates inhibitor. Of 255 LAC cases RI showed a sensitivity of $72 \%$ in correctly detecting LAC were as $11.7 \%$ were indeterminate \& $15 \%$ were wrongly interpretated as factor deficiency. SNR showed a sensitivity of $83 \%$ in correctly detecting LAC were as $10.9 \%$ were indeterminate \& $5.8 \%$ were wrongly interpretated as factor deficiency. Discussion: BSH \& ISTH guidelines recommends Standardised Normalised ratio (mixing test-specific cut off) and Rosners index (ICA) for interpretation of mixing test results in detecting LAC. This study shows that SNR is more sensitive than RI for detecting LAC while interpretating Mixing test results. This study was in correlation with Moore \& Kumano's study. Conclusion: It is difficult to interpretate mixing study results in LAC patients. It is valuable to maximise mixing test interpretation as the dilution can lead to false-negative results .RI \& SNR were comparatively analysed for their sensitivity to detect LAC in mixing studies \& these data applied with the reagents and equipment employed, SNR was found to be more sensitive as compared to RI.

Keywords: Activated partial thromboplastin time, antiphospholipid antibodies, antiphospholipid syndrome, diluted Russell's viper venom time, lupus anticoagulant.

Copyright @ 2019: This is an open-access article distributed under the terms of the Creative Commons Attribution license which permits unrestricted use, distribution, and reproduction in any medium for non-commercial use (NonCommercial, or CC-BY-NC) provided the original author and sources are credited.

SNR- Standard Normalised mixing ratio, APSAntiphospholipid antibody syndrome, LA- Lupus anticoagulant,

CLSI -Clinical and Laboratory Standards institute. dRVVT - diluted Russell's viper venom time

\section{INTRODUCTION}

The antiphospholipid antibody syndrome (APS) is a systemic, acquired, immune-mediated disorder characterized by episodes of venous, arterial, or microcirculation thrombosis and/or pregnancy abnormalities, associated with the persistent presence of autoantibodies, confirmed at least at two occasions 12 weeks apart and the antibodies directed to molecular complexes consisting of phospholipids and proteins [1].

Classification of APS (International consenses statement criteria) Miyakis et al. [2] 


\begin{tabular}{|c|c|}
\hline Clinical Criteria & Laboratory Criteria \\
\hline 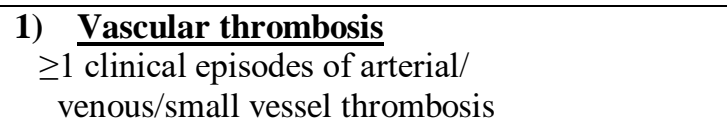 & $\begin{array}{l}\geq \mathbf{1} \text { or the following present in plasma on } 2 \text { occasions } \\
\text { more than } 12 \text { weeks apart. }\end{array}$ \\
\hline 2) Pregnancy morbidity & a) Lupus Anticoagulant (LA) \\
\hline $\begin{array}{l}\geq 3 \text { spontaneous miscarriages before 10th } \\
\text { week, not otherwise explained }\end{array}$ & $\begin{array}{l}\text { b) Anti-cardiolipin IgG or IgM Antibodies at med- } \\
\text { high titre ( }>99 \text { th centile) }\end{array}$ \\
\hline $\begin{array}{l}\geq 1 \text { unexplained death of a morphologically } \\
\text { normal fetus after the } 10 \text { th week }\end{array}$ & $\begin{array}{l}\text { c) Anti-B2-Glycoprotein-1 IgG or IgM Antibodies } \\
\text { at med-high titre ( }>99 \text { th centile) }\end{array}$ \\
\hline $\begin{array}{l}\geq 1 \text { pre-term birth of a morphologically normal } \\
\text { fetus before } 34 \text { th week due to eclampsia, pre- } \\
\text { eclampsia or placental insufficiency }\end{array}$ & \\
\hline
\end{tabular}

\section{Clinical + 1 Laboratory criteria required}

\section{Principle of testing for a LA}

1. Prolonged phospholipid-dependent clotting tests by two methods (e.g. DRVVT + Silica)

2. Demonstrate the presence of an inhibitor by use of a mixing study

3. Demonstrate the phospholipid dependence of the inhibitor (e.g. by use of high concentration Phospholipid)[1]

\section{Mixing Study Test Principle}

If PT and/or aPTT is prolonged then mixing test is indicated. A patient would generally need a level $\geq 40 \%$ of each factor that is being detected bythe test procedure to achieve a normal aPTT or PT test result. Therefore, a patient with an inadequate level, meaning less than $40 \%$, of one or more coagulation factor will have a prolonged PT or aPTT test. In the mixing study, an aliquot of abnormal patient plasma is mixed with an equal amount of pooled normal plasma (PNP), which contains approx. $100 \%$ of all coagulation factors. The new mixed plasma sample contains at least a $40 \%$ level of each factor after the mix, including the factors that may have been present in very low levels in the original sample $[2,3]$.
The Main Objective is to assess sensitivity of SNR vs RI in interpretation of Mixing tests in LAC cases retrospectively.

\section{MATERIALS \& METHODS Exclusion criteria \\ 1) DOACs \\ 2) Heparin or warfarin therapy \\ 3) factor $8 \& 9$ inhibitors \\ 4) factor deficiencies and VWD}

\section{Mixing Study Considerations Preanalytical variables}

- Clotted, hemolyzed, lipemic specimen

- Underfilled tube, wrong anticoagulant

- Must be platelet-poor, $<10,000 / \mathrm{uL}$

- $15 \%$ of anti-FVIII inhibitors are detected in immediate mix

- $15 \%$ of LAs require incubation

- Weak LAs may be missed in 1:1 mix

- $\quad$ Select a more LA-sensitive PTT reagent or prepare a 4:1 mix

Interpretation of mixing test

\begin{tabular}{|c|c|c|c|c|}
\hline Interpretation & Tube 1 (PCNP) & Tube 2 (PP) & $\begin{array}{c}\text { Tube } 3 \\
(1: 1 \text { PNCP:PP) }\end{array}$ & $\begin{array}{c}\text { Tube } 4 \\
(1: 1 \text { PNCP:PP) }\end{array}$ \\
\hline Incubate & $37^{0} \mathrm{C}$ for $2 \mathrm{hrs}$ & $37^{0} \mathrm{C}$ for $2 \mathrm{hrs}$ & $37^{0} \mathrm{C}$ for $2 \mathrm{hrs}$ & No incubation \\
\hline Normal Study & perform APTT & Perform APTT & perform APTT & Perform APTT immediately \\
\hline CF deficiency & Normal & Normal & Normal & Normal \\
\hline & Normal & APTT - Prolonged & Normal & Normal \\
\hline Factor VIII Inhibitor & Normal & APTT - Prolonged & APTT -Prolonged & \\
\hline (time dependent) & & & & APTT - \\
\hline Factor IX inhibitor & Normal & APTT - Prolonged & Normal & Prolonged \\
\hline (immediate acting) & & & & \\
\hline
\end{tabular}

The Coagulometer used is Sysmex CS-5100. APTT reagent is Pathrombin SL, LA1 (DRVVT) \& LA2 (confirmatory) reagents supplied by siemens.LA1/LA2 ratio > 1.15 is positive for LAC

\section{Manual calculation}

D R V V T Screen -

1. Pooled normal plasma + dilute phospholipid + DRVV + Calcium $\longrightarrow$ Clot time

2. Patient plasma + dilute phospholipid + DRVV +

Calcium $\rightarrow$ Clot time

3. Calculate ratio: (NR 0.9-1.05) 
Result \& Next Step

DRVVT Ratio >1.05 suggest possible LA
Mixing test NPP 1:1 PP (if weak LA suspected then NPP1:4 PP)

\section{Rosner Index = 1:1 mix PTT - PNP PTT $x$ 100. Cut offs $\leq 10=$ Correction $\& \geq 15=$ Inhibitor. patient PT}

SNMR is derived from the upper limit of population distribution data for screening test ratios performed on 1:1 mixtures with common normal pooled plasma.

SNMR = 1:1 mix sample (seconds)/ 1:1 mix reference interval mean (seconds)[4-6]

\section{$\%$ Correction}

$\%$ Correction calculated following a neutralization step when extra phospholipid (+PL) is added and the DRVVT repeated.

(Patient DRVVT / Control DRVVT) - (Patient DRVVT+PL / Control DRVVT +PL) Test DRVVT / Control DRVVT

A positive correction of $>10 \%$ is considered consistent with a lupus anticoagulant.

\section{APT T}

1. SCT Screen ratio $=$ Patient SCT Screen $/$ Mean of SCT Screen Reference Range

2. SCT Confirm ratio $=$ Patient SCT Confirm $/$ Mean of SCT Confirm Reference Range

3. Normalised ratio $=$ Screen ratio $/$ Confirm ratio

An increased normalised ratio suggests presence of a lupus anticoagulant ( $>1.16$ or $>1.24$ depending on analyser and reagents used) [7].

\section{RESULTS}

A total 255 LAC + ve cases were retrospectively choosen as per the exclusion criteria. Screen and confirm dRVVT and dilute APTT assays were performed on undiluted plasma and 1:1 mixtures with normal pooled plasma. All the cases were either DRVVT positive or SAPTT or both \& were positive for screening \& confirmatory tests

Table-1: Mixing test detecting Inhibitor

\begin{tabular}{|l|l|}
\hline Mixing test (255) & \\
\hline RI (ICA) > 15 (No Correction) & $185(72 \%)$ \\
\hline SNR > 1.15 (No correction) & $212(83 \%)$ \\
\hline & \\
\hline
\end{tabular}

Table-2: Mixing test interpretated as indeterminate

\begin{tabular}{|l|l|}
\hline Mixing test & $30(11.7 \%)$ \\
\hline $\begin{array}{l}\text { RI (ICA ) }=11-15 \\
\text { (indeterminate) }\end{array}$ & $28(10.9 \%)$ \\
\hline $\begin{array}{l}\text { SNR }=1.1-1.4 \\
\text { (indeterminate) }\end{array}$ & \\
\hline & \\
\hline
\end{tabular}

Table-3: Mixing test interpretated as factor deficiency

\begin{tabular}{|l|l|}
\hline Mixing test & \\
\hline RI (ICA ) $=<10$ (Correction) & $40(15.6 \%)$ \\
\hline SNR = <1 ( Correction) & $15(5.8 \%)$ \\
\hline & \\
\hline
\end{tabular}

RI showed a sensitivity of $72 \%$ in correctly detecting LAC were as $11.7 \%$ were indeterminate \& $15 \%$ were wrongly interpretated as factor deficiency.

SNR showed a sensitivity of $83 \%$ in correctly detecting LAC were as $10.9 \%$ were indeterminate \& $5.8 \%$ were wrongly interpretated as factor deficiency

\section{DISCUSSION}

The antiphospholipid antibody syndrome (APS) is a systemic, acquired, immune-mediated disorder characterized by episodes of venous, arterial, or microcirculation thrombosis and/or pregnancy abnormalities, associated with the persistent presence of autoantibodies, confirmed at least at two occasions 12 weeks apart and the antibodies directed to molecular complexes consisting of phospholipids and proteins [1].

APS cause preeclampsia (18\%), pregnancyinduced hypertension, foetal death $(7 \%)$, retardation $(31 \%)$, premature labor $(43 \%)$, stillbirth, and ultimately sterility [1].

The Current 3 major LAC guidelines (BSH, ISTH \& CLSI) recommends mixing tests for detection of LAC, even though these test order/sequence vary \& there are certain limitations, but still these guidelines advocates mixing test so as to maximize the diagnostic performance.

\section{INTERPRETATION OF MIXING STUDIES RESULTS [4]}

1) If results of Mixing study shows correction for both the immediate \& incubated APTT, the patient most likely has a single/multiple factor deficiencies

2) If Mixing study results shows no correction in either immediate or incubated APTT, the patient may have a coagulation inhibitor most likely LAC

3) If mixing test results shows correction for immediate APTT, but no correction for incubated APTT, the patient may have a slow acting inhibitor such as factor VIII 
My previous article was on comparison of RI vs Changs \% correction in interpretation of Mixing study. RI with a cut off value of $<10$ is $92.5 \%$ sensitive in diagnosing Factor deficiency $\&$ a cut off value of $>15$ is $91.1 \%$ sensitive for inhibitor diagnosis \& it could not categorise, $8 \%$ of total cases into factor deficiency /inhibitor.

RI showed a sensitivity of $72 \%$ in correctly detecting LAC were as $11.7 \%$ were indeterminate \& $15 \%$ were wrongly interpretated as factor deficiency.
SNR showed a sensitivity of $83 \%$ in correctly detecting LAC were as $10.9 \%$ were indeterminate \& $5.8 \%$ were wrongly interpretated as factor deficiency

Our study shows that SNR is more sensitive than RI for detecting LAC while interpretating Mixing test results. This study was in correlation with Moore \& Kumano's study titled Mixing test specific cut-off is more sensitive at detecting lupus anticoagulants than index of circulating anticoagulant.

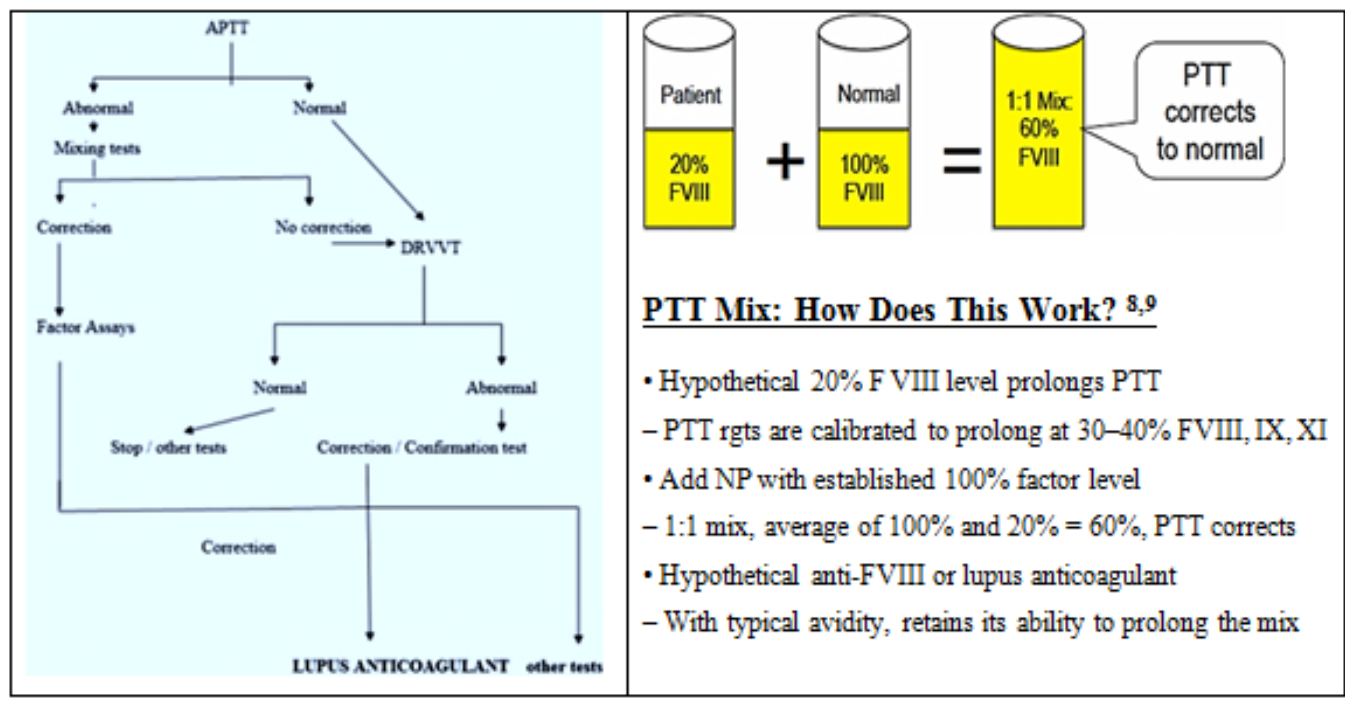

Diagnostic algorithm for LAC

\section{What Limit Defines Correction?[10-12]}

\section{Limits based on a fixed PTT value such as reference interval}

- 1:1 mix within RI upper limit $(95 \%$ or $99 \%$ confidence interval, 39\%)

- 1:1 mix within RI upper limit +5 seconds $(8 \%)$

\section{Limits based on the pooled normal plasma PTT} value

- $1: 1$ mix within NP PTT value +5 seconds $(14 \%)$

- $1: 1$ mix within NP PTT + 10\% (32\%)

\section{Rosner or Chang limit formula using patient, NP,} and $1: 1 \mathrm{mix}$ results

- Rosner formula produces a ratio

- Chang's formula produces \% deviation, requires incubation of patient plasma

\section{CONCLUSION}

It is difficult to interpretate mixing study results in LAC patients. It is valuable to maximise mixing test interpretation as the dilution can lead to false-negative results .RI \& SNR were comparatively analysed for their sensitivity to detect LAC in mixing studies \& these data applied with the reagents and equipment employed, SNR was found to be more sensitive as compared to RI.

\section{REFERENCES}

1. Wendy, L. (2009). Antiphospholipid Antibody Syndrome. ASH Education Book, 1(1):233-239.

2. Miyakis, S., Lockshin, M. D., Atsumi, T., Branch, D. W., Brey, R. L., Cervera, R. H. W. M., ... \& Reber, G. (2006). International consensus statement on an update of the classification criteria for definite antiphospholipid syndrome (APS). Journal of Thrombosis and Haemostasis, 4(2), 295-306.

3. Moore, G. W., Culhane, A. P., Daw, C. R., Noronha, C. P., \& Kumano, O. (2016). Mixing test specific cut-off is more sensitive at detecting lupus anticoagulants than index of circulating anticoagulant. Thrombosis research, 139, 98-101.

4. D'Angelo, G. (2019). Laboratory Methodologic Approach in Prolonged Activated Partial Thromboplastin Time Test. Modern Medical Laboratory Journal, 3(1), 39-43.

5. Rosner, E., Pauzner, R., Lusky, A., Modan, M., \& Many, A. (1987). Detection and quantitative evaluation of lupus circulating anticoagulant activity. Thrombosis and haemostasis, 57(02), 144147.

6. Chang, S. H., Tillema, V., \& Scherr, D. (2002). A "percent correction" formula for evaluation of mixing studies. American journal of clinical pathology, 117(1), 62-73. 
7. Ali, F., Bhagavathi, S., \& Mattson, J. (2006, October). Evaluation and comparison of mixing study results using percent correction formulae (Chang vs Rosner indices) to optimize and standardize the reporting of mixing study results. In american journal of clinical pathology (Vol. 126, No. 4, pp. 638-638). 2100 W HARRISON ST, CHICAGO, IL 60612 USA: AMER SOC CLINICAL PATHOLOGY.

8. Nichols, W. L., Kottke-Marchant, K., LedfordKraemer, M. R., Homburger, H. A., \& Cardel, L. K. (2012). Lupus anticoagulants, antiphospholipid antibodies, and antiphospholipid syndrome. Laboratory hematology practice. West Sussex, UK: Wiley-Blackwell Publishing ltd, 50925.
9. Brandt, J. T., Triplett, D. A., Alving, B., \& Scharrer, I. (1995). Criteria for the diagnosis of lupus anticoagulants: an update. Thrombosis and haemostasis, 74(04), 1185-1190.

10. British Journal of Haematology. (2013).160:153170.

11. Verbruggen, B., Dardikh, M., Polenewen, R., Van Duren, C., \& Meijer, P. (2011). The factor VIII inhibitor assays can be standardized: results of a workshop. Journal of thrombosis and haemostasis, 9(10), 2003-2008.

12. Bain, B. J., Bates, I., Laffan, M. A., \& Lewis, S. M. (2016). Dacie and Lewis Practical Haematology: Expert Consult: Online and Print. Elsevier Health Sciences. 